\title{
EFECTIVIDAD DE LAS TERAPIAS PSICOLÓGICAS: UN ANÁLISIS DE LA REALIDAD ACTUAL
}

\author{
ENRIQUe ECHEBURÚA, PAZ DE CORRAL y KARMEle SALABERRÍA \\ Facultad de Psicología, Universidad del País Vasco, San Sebastián
}

\begin{abstract}
Resumen: En los últimos años las terapias psicológicas empíricamente validadas han experimentado un gran desarrollo en diversas áreas. Los pacientes buscan actualmente ayuda terapéutica no sólo por los trastornos mentales tradicionales. La psicología clínica basada en la evidencia implica la integración de la experiencia clínica con los resultados de la investigación. Sin embargo, hay muchas discrepancias entre la investigación y la práctica clínica: no siempre un tratamiento eficaz en un ensayo clínico resulta efectivo en la práctica clínica habitual. Además, hay algunas limitaciones para generalizar los logros de la investigación a la práctica clínica habitual y, por ello, su difusión a los profesionales clínicos es aún muy limitada. Se abordan asimismo cuestiones éticas relevantes en relación con la práctica clínica y se analizan las características psicológicas del terapeuta que están más relacionadas con el éxito terapéutico. Por último, se señalan algunos retos de futuro y se plantean nuevas líneas de investigación.
\end{abstract}

Palabras clave: Tratamientos empíricamente validados; limitaciones; dificultades de difusión; cuestiones éticas.

\section{Effectiveness of psychological therapies: A review of the current situation}

\begin{abstract}
In recent years evidence-based psychological treatments have evolved greatly in several areas. Patients are now seeking therapeutic assistance not only for traditional mental disorders. Evidence-based clinical psychology implies integrating clinical expertise with research evidence. However, there are many discrepancies between research and clinical practice: a treatment proved efficacious in a study is not always efficient in ordinary clinical practice. Furthermore, there are limitations to generalizing research results to ordinary clinical practice, so their acceptance by clinical professionals is still quite limited. Relevant ethical questions related to clinical practice are considered, and psychological characteristics of the therapist that have a bearing on therapeutic success are analyzed. Finally, some future challenges are named and new lines of research are proposed.
\end{abstract}

Keywords: Empirically validated treatments, limitations, difficulties of dissemination, ethical questions.

\section{INTRODUCCIÓN}

La preocupación reciente por las terapias efectivas no es ajena a los cambios sociales actuales. La contención del gasto vigente en la Sanidad Pública y en las compañías de seguros ha impulsado en los últimos años la investiga-

Recibido: 23-marzo-2010; Aceptado: 25-marzo-2010

Correspondencia: Enrique Echeburúa, Universidad del País Vasco, Facultad de Psicología, Avda. de Tolosa 70, 20018 San Sebastián

Correo-e: enrique.echeburua@ehu.es ción sobre la efectividad de las terapias psicológicas. Al margen de los posibles excesos cometidos por un enfoque meramente economicista de la salud, no cabe duda de que esta orientación ha supuesto un giro radical en la investigación sobre los tratamientos psicológicos. Ya no se trata sólo de demostrar que una terapia es eficaz para un determinado trastorno, sino que lo es más que otras alternativas y en unas mejores condiciones (más breve, en un régimen ambulatorio, etcétera). No es razonable mantener, especialmente en los centros públicos, terapias que no han mostrado ser eficaces más allá de un 
Tabla 1. ¿Qué quiere decir “eficacia del tratamiento”? (Marks y O’Sullivan, 1992)

\begin{tabular}{|c|c|}
\hline Componentes de la eficacia & Significado \\
\hline Especificidad & ¿Qué síntomas mejoran? \\
\hline Intensidad & ¿Cuánto mejoran los síntomas? \\
\hline Plazo & ¿Cuánto tarda en comenzar la mejoría? \\
\hline Duración a corto plazo & ¿Dura el efecto terapéutico mientras el tratamiento se mantiene? \\
\hline Duración a largo plazo & ¿Continúa el efecto tras la interrupción del tratamiento? \\
\hline Costes & Rechazos, efectos secundarios y abandonos \\
\hline Interacciones & ¿Cómo interactúa con otros tratamientos? \\
\hline Balance & ¿Cuáles son las ventajas e inconvenientes en relación con otros tratamientos disponibles? \\
\hline
\end{tabular}

efecto placebo o de los efectos inespecíficos de cualquier terapia (Bayés, 1984; Echeburúa y Corral, 2001).

Sin embargo, el concepto de eficacia terapéutica dista mucho de ser unívoco. Resulta sorprendente la poca atención que se ha prestado en la bibliografía relacionada con las investigaciones clínicas a este aspecto y a los equívocos suscitados por las distintas interpretaciones de dicho término (Echeburúa, Corral y Salaberría, 1998). Cuando se habla de los efectos de un determinado tratamiento - sea éste psicofarmacológico, psicológico o combinado-, tal término puede tener diversos significados, tal como se expone en la Tabla 1.

En los últimos veinte años se han producido tres avances muy importantes en el campo de los trastornos mentales. En primer lugar, se han hecho grandes adelantos en la comprensión de las bases biológicas de muchos cuadros clínicos (la esquizofrenia, la depresión o el trastorno bipolar, por ejemplo). En segundo lugar, se ha desarrollado una nueva generación de psicofármacos (antidepresivos y neurolépticos especialmente), con una mayor eficacia terapéutica y un perfil más favorable de efectos secundarios. Y en tercer lugar - pero no menos importante-, se han creado tratamientos psicológicos breves y efectivos para una amplia variedad de trastornos (Bados, García y Fuste, 2002).
Sin embargo, la difusión de estos tres avances no ha sido simétrica. En los dos primeros casos la industria farmacéutica se ha ocupado de difundirlos ampliamente mediante diversas publicaciones gratuitas (folletos, revistas, libros, etcétera) y congresos financiados. Por el contrario, en el tercer caso - el desarrollo de los tratamientos psicológicos-, al no contar con un órgano de difusión tan poderoso como la industria farmacéutica (que, por motivos obvios, no está interesada en este tema), los avances habidos se han limitado a las revistas científicas y no han llegado suficientemente a los sectores profesionales implicados (Echeburúa, 1998). De hecho, los tratamientos psicofarmacológicos (especialmente en el caso de los antidepresivos y de los neurolépticos) prescritos en los centros clínicos han cambiado considerablemente en los últimos años al hilo de los nuevos descubrimientos, pero, sin embargo, los avances en las terapias psicológicas no se han reflejado en la práctica clínica habitual ni siquiera a veces en los contenidos formativos de los programas de psicología clínica (Echeburúa, Corral y Salaberría, 2005).

En este artículo se aborda el tema de las terapias empíricamente validadas y de las nuevas tendencias, pero también se señalan sus limitaciones. Y también se hace referencia a algunos temas menos de moda (pero, sin embargo, extraordinariamente relevantes), como son los aspectos deontológicos implicados en la prácti- 
ca profesional o el análisis de los requisitos psicológicos necesarios (o, al menos, convenientes) para el ejercicio profesional de la psicología clínica.

\section{LAS NUEVAS DEMANDAS TERAPÉUTICAS}

Las demandas terapéuticas han cambiado considerablemente en los últimos años. Ahora se tiende a consultar, además de por los cuadros clínicos tradicionales (depresión, trastornos de ansiedad, esquizofrenia, trastornos de la conducta alimentaria o adicciones), por problemas menores, que, sin constituir propiamente trastornos mentales, reflejan una patología del sufrimiento o de la infelicidad. Entre ellos se encuentran el duelo por la pérdida de un ser querido, los conflictos de pareja o la ruptura de pareja no deseada, las dificultades de convivencia y educación con los hijos, las conductas violentas de los adolescentes, los problemas de estrés laboral, el sufrimiento de los inmigrantes, el dolor crónico, el cansancio por el cuidado de personas dependientes, las consecuencias psicológicas del daño cerebral en el enfermo y su familia, etcétera.

En las clasificaciones psiquiátricas estos problemas adaptativos se denominan códigos $Z$ y son un reflejo de las dificultades de la vida cotidiana, así como de los efectos de la soledad y de la incomunicación que prevalecen en nuestra sociedad. Y el volumen de las consultas de este tipo puede suponer casi el $20 \%$ de las demandas asistenciales en los centros de salud mental.

Estas nuevas demandas terapéuticas están relacionadas con una mayor exigencia de calidad de vida por parte de los pacientes y con una mayor intolerancia al malestar emocional, pero también con la medicalización de la vida cotidiana. De hecho, hay una tendencia malsana por asignar a todos los problemas un nombre clínico, unos síntomas y un tratamiento: es una tendencia que se ve acentuada por el marketing de la industria farmacéutica dirigido a médicos y pacientes. No deja de ser significativo, por ejemplo, que la primera edición del DSM (DSM-I, 1952) contenía unos 60 trastornos mentales y que la actualmente vigente (DSM-IV-TR, 2000) recoja más de 300 . No parece razonable pensar que en menos de cincuenta años los trastornos mentales se hayan multiplicado por cinco. Por ello, hay que estar precavido ante los supuestos nuevos diagnósticos clínicos: síndrome posvacacional, adicción a las compras, síndrome de Diógenes, vigorexia, síndrome de alienación parental, síndrome de Ulises, etcétera.

En resumen, muchas de las consultas a los psicólogos clínicos hoy no se relacionan con trastornos mentales, sino con situaciones de infelicidad y sufrimiento psicológico. Se trata de personas que se sienten sobrepasadas en sus estrategias de afrontamiento para las dificultades cotidianas y que, muy frecuentemente, carecen de una red de apoyo familiar y social sólida y estable que les proporcione la ayuda y el apoyo necesario para seguir adelante. Los psicólogos clínicos tienen que adaptarse a esta nueva realidad, evitar la tendencia a establecer diagnósticos psiquiátricos y desarrollar unas estrategias de intervención (técnicas de counseling o de intervención en crisis) que no son exactamente las mismas que han mostrado éxito en el tratamiento de los trastornos mentales propiamente dichos. Y probablemente el lugar más adecuado para detectar y tratar estos problemas no sean los centros de salud mental, sino los centros de atención primaria, en donde resulta absurdo reducir el sufrimiento a categorías diagnósticas. Éste es un reto de futuro importante.

\section{¿POR QUÉ HAY QUE EVALUAR LA EFICACIA DE LAS TERAPIAS?}

La evaluación de la eficacia de las intervenciones terapéuticas es una necesidad perentoria. En primer lugar, porque el progreso científico de la psicología clínica requiere la delimitación de las terapias eficaces, así como de los componentes activos de las mismas. En segundo lugar, porque esta disciplina se enfrenta, como fruto de su propio desarrollo, a retos de cada vez mayor complejidad (por ejemplo, al tratamiento de los trastornos de personalidad, de los problemas de la conducta alimentaria o de las nuevas adicciones). $\mathrm{Y}$ en tercer lugar, porque las 
terapias psicológicas están ya incorporadas a los centros de salud mental públicos. De este modo, la Sanidad Pública sólo debe ofertar terapias efectivas $\mathrm{y}$, en igualdad de condiciones, breves (Barlow y Hoffman, 1997). Es decir, acortar el sufrimiento del paciente y ahorrar gastos y tiempo a los centros parecen objetivos prioritarios (Bayés, 1984; Echeburúa, 1998; Guimón, 2004).

Por ello, las ventajas de la psicología clínica basada en la evidencia son claras. Los organismos públicos y las compañías de seguros pueden contar con criterios operativos para financiar selectivamente sólo las terapias psicológicas empíricamente validadas. A su vez, los pacientes pueden contar con una información clara para saber qué tipo de ayuda buscar para hacer frente a sus problemas psicológicos; y los profesores universitarios, con una orientación clara para formar a los futuros psicólogos en aquellas terapias que han mostrado su utilidad. Finalmente, desde una perspectiva deontológica o judicial, se puede definir operativamente el concepto de mala praxis (cuando el terapeuta se aparta de una práctica profesional empíricamente validada para un trastorno concreto y en unas circunstancias determinadas).

La psicología clínica basada en la evidencia ha contribuido también a delimitar la duración de los tratamientos efectivos. En general, con una terapia psicológica entre 8 y 24 sesiones se obtiene, según los cuadros clínicos de que se trate, una mejoría del 70\% (Turner, Beidel, Spaulding y Brown, 1995). Prolongar la terapia más allá de 24 sesiones y alargarla a 12 meses aumenta muy poco el porcentaje de mejoría e incrementa enormemente el gasto (Figura 1). Huelga decir que el psicoanálisis, que cuenta con 600 a 1000 sesiones por término medio, queda fuera por completo de este contexto. Otra cuestión son las psicoterapias psicoanalíticas de menor duración o de tiempo limitado, que en algunos estudios han mostrado su eficacia (Leichsenring y Leibing, 2003).

\section{PSICOLOGÍA CLÍNICA BASADA EN LA EVIDENCIA}

La psicología clínica basada en la evidencia es aquella que se aplica tanto en la comprensión como en el diagnóstico y en el tratamiento de los distintos trastornos métodos que han sido contrastados científicamente. Este enfoque de trabajo supone un acercamiento empírico y un espíritu de mejora y autocrítica continua en beneficio de los pacientes, que cuestiona continuamente la labor que realizamos los psicólogos clínicos y que nos hace ser precavidos en nuestras afirmaciones.

Así, cuando alguien afirma que un tratamiento es eficaz, hay que analizar en qué tipo

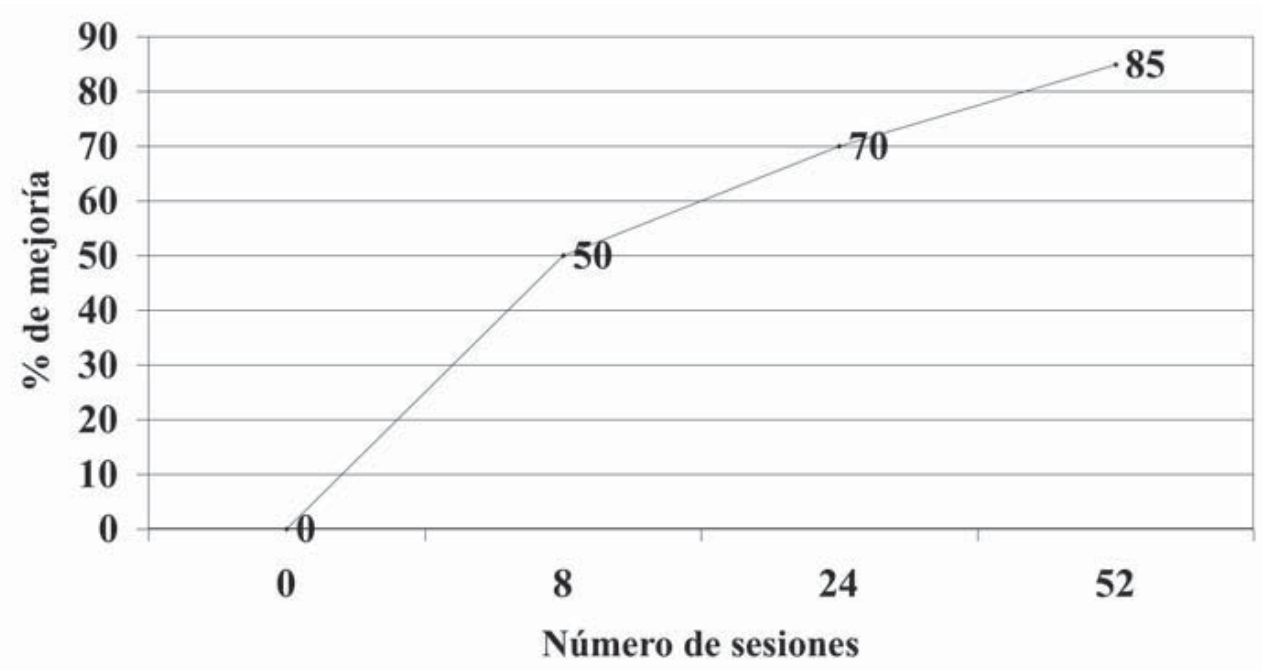

Figura 1. Relación entre el número de sesiones y los efectos terapéuticos (Howard et al., 1986). 
Tabla 2. Niveles de evidencia

\begin{tabular}{cl}
\hline Nivel de evidencia & \multicolumn{1}{c}{ Tipo de estudio } \\
\hline Mínima & \\
& - Descripción de casos únicos \\
& - Estudios descriptivos o resoluciones de comités de expertos \\
& - Estudios de caso controlados \\
& - Ensayos controlados bien diseñados, pero no aleatorizados, o estudios naturalistas \\
& - Ensayos controlados y aleatorizados con muestras pequeñas \\
Máxima &
\end{tabular}

de pruebas se basa para afirmarlo. Hay diversos niveles de evidencia (Tabla 2). Actualmente se considera que el mayor nivel de evidencia está recogido en los estudios meta-analíticos de ensayos controlados y aleatorizados.

Sin embargo, muchos terapeutas no actualizan la información, bien porque no tienen acceso a ella o bien porque, simplemente, se resisten a cambiar sus métodos tradicionales. La Colaboración Cochrane, creada en 1993 en el Reino Unido, pretende dotar a médicos y pacientes de las herramientas necesarias para que todo tratamiento médico esté sustentado por la evidencia científica. En líneas generales, de lo que se trata es de revisar por medio del metaanálisis los estudios disponibles sobre enfermedades y sus tratamientos para evitar una variabilidad excesivamente grande de la práctica profesional y fomentar una toma de decisiones adecuada (Gambrill, 1999).

Por lo que se refiere específicamente a la psicología clínica, en los quince últimos años ha habido un intento riguroso - el más serio de los emprendidos hasta el momento - para evaluar la eficacia de las terapias psicológicas. Ya no se trata de determinar la validez de las psicoterapias en su conjunto o como aplicación a problemas psicológicos inespecíficos, como se había hecho en las últimas décadas (por ejemplo, Smith, Glass y Miller, 1980), sino de evaluar tratamientos eficaces para trastornos concretos en muestras clínicas claramente especificadas (Barlow, 1994; Chambless y Hollon, 1998).
A iniciativa del Congreso de Estados Unidos, se creó en 1989 una agencia federal - la Agency for Health Care Policy and Researchque tiene como objetivo determinar la eficacia de los tratamientos psicológicos para los trastornos mentales y establecer un directorio de las terapias de eficacia probada, con la finalidad última de mejorar la calidad del sistema de salud. Las guías elaboradas sirven, además, como criterio de financiación para las compañías aseguradoras, en cuanto al tipo y duración de una terapia, y para resolver demandas judiciales en relación con una práctica profesional supuestamente inadecuada. Se trata asimismo de orientar a los usuarios y de dotar de criterios a los responsables de los Servicios de Salud Mental para promover en los Centros Públicos sólo aquellas terapias que estén validadas empíricamente (Chambless, 1996).

En la elaboración de este informe, auspiciado por la División 12 (Psicología Clínica) de la Asociación Psicológica Americana y dirigido por Chambless (Task Force on the Promotion and Dissemination of Psychological Procedures, 1995) y revisado hasta la fecha en varias ocasiones (Chambless et al., 1996; Chambless et al., 1998), la determinación de la eficacia de un tratamiento viene avalada por la presencia de tres criterios: a) estar respaldado por la existencia de dos o más estudios rigurosos de investigadores distintos con diseños experimentales intergrupales; b) contar con un manual de tratamiento claramente descrito; y c) haber sido puesto a prueba en una muestra de pacientes 
Tabla 3. Requisitos convenientes en los ensayos clínicos sobre la eficacia de tratamientos (modificado de Seligman, 1995)

- Asignación aleatoria de los pacientes (no sujetos análogos) a las condiciones experimentales y de control. Mínimo de 30 sujetos por cada modalidad terapéutica.

- Evaluación detallada con arreglo a criterios diagnósticos operativos del DSM-IV. Medidas de evaluación múltiples.

- Entrevistadores ciegos respecto al grupo de tratamiento asignado al paciente evaluado.

- Ensayo clínico concurrente (los tratamientos en las distintas condiciones se aplican al mismo tiempo) y prospectivo (proyectado de ahora en adelante, no con datos anteriores).

- Exclusión de pacientes con trastornos múltiples.

- Tratamientos claramente descritos y estandarizados.

- Fijación de un número determinado de sesiones.

- Seguimiento de los pacientes a largo plazo (al menos, 12 meses).

inequívocamente identificados (por ejemplo, con arreglo a los criterios diagnósticos del DSM-IV) (Hickey, 1998). Más en concreto, las condiciones necesarias para un ensayo clínico figuran agrupadas en la Tabla 3 (Seligman, 1995).

De modo complementario, Chambless y Hollon (1998) se han referido a la existencia de tres tipos de eficacia: a) tratamiento eficaz $y$ especifico: aquel que es mejor que un tratamiento alternativo o que un placebo; b) tratamiento eficaz: aquel que es mejor que la ausencia de terapia en, al menos, dos estudios independientes y c) tratamiento probablemente eficaz: aquel que obtiene resultados positivos pero que no ha sido replicado todavía.

En diversas publicaciones recientes (Echeburúa y Corral, 2001; Mace, Moorey y Roberts, 2005; Nathan y Gorman, 1998; Pérez, Fernández-Hermida, Fernández y Amigo, 2003) figura una lista actualizada de los tratamientos psicológicos apoyados empíricamente para trastornos específicos, que está sujeta a revisiones periódicas. Se trata de una verificación empírica (experimental) y no meramente clínica (subjetiva), con una referencia clara al coste/eficacia. De esta lista se pueden obtener varias conclusiones: a) la superioridad de las terapias cognitivo-conductuales como tratamientos de elección; y b) la ausencia generalizada de las terapias psicodinámicas, a excepción de la terapia interpersonal para la depresión y la bulimia y quizá algunas intervenciones psicodiná- micas breves. El tema no está, en modo alguno, cerrado. Hay trastornos para los cuales no existen todavía tratamientos eficaces y específicos (por ejemplo, los trastornos psicóticos y los trastornos de personalidad) y no se han estudiado suficientemente los fracasos del tratamiento, es decir, de qué modo predecirlos y qué alternativas terapéuticas plantear en esos casos (Wilson, 1996a).

Al margen de las limitaciones de este enfoque, no deja de ser sorprendente que muchos psicólogos clínicos en ejercicio no estén adiestrados en este tipo de tratamientos y que muchos programas de formación en psicología clínica se hagan aún hoy a espaldas de estos conocimientos (Barlow y Hoffman, 1997).

\section{LIMITACIONES DE LAS INVESTIGACIONES CLÍNICAS}

Las terapias empíricamente validadas han concedido una gran importancia a la clasificación de los trastornos mentales. Esta preocupación por el enfoque científico ha llevado a sobrevalorar el objeto de la psicología (los cuadros clínicos) y a desatender, al menos hasta cierto punto, al sujeto de la psicología clínica (el ser humano sufriente). Es decir, la importancia concedida al diagnóstico clínico (por ejemplo, tratamientos efectivos para el trastorno obsesivo-compulsivo) ha diluido la atención específica al paciente que padece esa patología 
y no ha tomado en consideración las diferencias individuales existentes entre los pacientes catalogados con el mismo diagnóstico. En este sentido, hay una tendencia a una cierta cosificación del paciente. De este modo, los manuales o guías de tratamiento pueden, a veces, responder (si no se usan bien) a la mentalidad del libro de recetas de cocina (Becoña et al., 2004).

La psicología clínica basada en la evidencia está sujeta a diversas limitaciones: las diferencias de rendimiento de los terapeutas con una misma terapia, la variación de la gravedad de los pacientes con un mismo diagnóstico o la inexistencia de pacientes con un único diagnóstico puro (la comorbilidad es la norma, no la excepción), sin olvidar que distintos tipos de tratamientos pueden ser igualmente efectivos en un mismo cuadro clínico (sólo que para distintos perfiles de pacientes).

Las investigaciones clínicas, dotadas de una gran validez interna, permiten determinar el alcance terapéutico de un programa de intervención, es decir, su grado de eficacia. De este modo, se puede saber si los cambios observados en la variable dependiente son atribuibles al tratamiento y no a otras circunstancias (paso del tiempo, remisión espontánea, etcétera). Otra cosa es, sin embargo, la efectividad de dicho programa en la práctica clínica habitual, que es lo que le confiere la validez externa o ecológica. Un tratamiento deja de ser eficaz para convertirse en efectivo cuando se pueden generalizar los resultados obtenidos en la investigaciónsometida a un riguroso control y en condiciones óptimas - a las situaciones clínicas reales (Becoña, 1999). Por último, la eficiencia, que no puede limitarse a una mera contención del gas- to, hace referencia al logro de los objetivos terapéuticos con el menor coste posible (en términos de dinero, tiempo del terapeuta o sufrimiento del paciente) (Turner et al., 1995) (Figura 2). De hecho, la terapia psicológica puede implicar una reducción considerable de costes a largo plazo cuando se utiliza aislada o en combinación con un tratamiento farmacológico en muchos trastornos mentales, como la ansiedad y la depresión, e incluso en trastornos graves, como la esquizofrenia, el trastorno bipolar o el trastorno límite de personalidad (Gabbard, Lazar, Hornberger y Spiegel, 1997; Layard, 2006).

La relación coste/beneficio no entraña un criterio meramente economicista. Se trata de determinar que los resultados justifican una inversión a nivel terapéutico (coste de la terapia), a nivel sanitario (ahorro de tratamientos ulteriores) y a nivel social (menor absentismo laboral, menor uso de los recursos sociales, prevención de problemas en otros miembros de la familia, etcétera). No hay que confundir, sin embargo, la eficiencia con una mera reducción del gasto. Así como en la eficiencia se trata de obtener el máximo beneficio de los recursos disponibles, la reducción del gasto trata simplemente de abaratar los servicios ofrecidos, con una preocupación máxima en el ahorro y mínima en la calidad. El economicismo vigente en la sanidad pública actual refleja probablemente más la preocupación por la reducción de costes y del gasto que un interés genuino por la eficiencia (Labrador, Echeburúa y Becoña, 2000).

Hay también otras limitaciones de las investigaciones clínicas. En la investigación los pa-

\begin{tabular}{lll}
\hline Validez Interna & $\begin{array}{l}\text { Resultados favorables en un } \\
\text { contexto de investigación }\end{array}$ \\
\cline { 2 - 4 } & Efectividad & $\begin{array}{l}\text { Resultados favorables en la } \\
\text { práctica clínica habitual }\end{array}$ \\
\hline Eficiencia & $\begin{array}{l}\text { Buenos resultados al menor } \\
\text { coste posible }\end{array}$ & Validez externa \\
\hline
\end{tabular}

Figura 2. Criterios de éxito en la terapia (Echeburúa y Corral, 2001). 
cientes son homogéneos entre sí (en cuanto a edad y sexo, por ejemplo) y puros, sin presentar comorbilidad con otros trastornos, y los terapeutas, que no suelen tener una gran experiencia clínica y que el paciente no puede elegir, están muy motivados. A su vez, los tratamientos, más bien cortos, se ajustan a una guía rígida y a un diario de sesiones. Por el contrario, en la práctica clínica habitual los pacientes están aquejados de varios trastornos simultáneamente, son heterogéneos y han sido sometidos, habitualmente, a tratamientos previos; los terapeutas cuentan con una experiencia clínica amplia y son objeto de elección por parte del paciente, al menos en la práctica privada; $y$, por último, los tratamientos son más largos y flexibles: no hay un número limitado de sesiones, el contenido del tratamiento es flexible y está sujeto a cambios. En definitiva, no hay un isomorfismo entre la investigación y la clínica (Tabla 4).

Respecto a la interpretación de los resultados de una investigación, no se debe confundir la significación estadística de una investigación con la significación clínica. De este modo, y al margen de que el control de los errores de tipo I y de tipo II en la significación estadística requiere el cálculo de la intensidad del cambio mediante el tamaño del efecto (que sólo es adecuado cuando está por encima de $0,7 / 0,8)$, la significación clínica se calcula mediante las puntuaciones de cambio. En concreto, un paciente se considera significativamente mejorado sólo si en el postratamiento o en el seguimiento está por debajo de 2 desviaciones típicas de la media de la mues- tra en el pretratamiento (Pascual, Frías y Monterde, 2004; Salaberría, Páez y Echeburúa, 1996). Además hoy día los resultados obtenidos por los pacientes en una investigación pueden ser comparados con baremos de la población general y de este modo se puede comprobar si los pacientes pueden considerarse recuperados o no.

Por lo que se refiere al desarrollo de la investigación, no hay una correspondencia directa entre los logros de la psicopatología experimental y los resultados del tratamiento. Hay hoy un conocimiento más detallado de los procesos cognitivos implicados, por ejemplo, en los trastornos de ansiedad o en la depresión, pero no por ello ha habido un avance terapéutico claro del que puedan beneficiarse los pacientes. Esta última reflexión, sin llevar consigo una carga pesimista, trata de salir al paso de un cierto triunfalismo que se regodea en avances científicos sin repercusión clínica.

Por último, la organización de los centros de salud mental, al nivel de la asistencia pública, puede constituir una rémora para la aplicación efectiva de las terapias empíricamente validadas. Si bien la primera visita se hace en un tiempo corto para no engrosar la lista de espera (y salvar de este modo las estadísticas oficiales), las siguientes visitas pueden tener un carácter mensual o bimestral por la presión asistencial existente. De este modo, el tratamiento psicológico se aplica en una infradosis terapéutica y se caracteriza más por un seguimiento que por un tratamiento propiamente dicho. No es por ello infrecuente dar el alta una vez remitida la fase

Tabla 4. Diferencias entre la investigación y la práctica clínica (Echeburúa y Corral, 2001)

\begin{tabular}{|c|c|c|}
\hline VARIABLES & INVESTIGACIÓN & PRÁCTICA CLÍNICA \\
\hline Pacientes & $\begin{array}{l}\text { - Homogéneos. } \\
\text { - Sin comorbilidad. }\end{array}$ & $\begin{array}{l}\text { - Heterogéneos. } \\
\text { - Con diversos trastornos. }\end{array}$ \\
\hline Terapeutas & $\begin{array}{l}\text { - Muy motivados, pero con poca } \\
\text { experiencia. }\end{array}$ & $\begin{array}{l}\text { - Con menor motivación, pero con mayor experiencia } \\
\text { profesional. }\end{array}$ \\
\hline Tratamiento & $\begin{array}{l}\text { - Contenido rígido. } \\
\text { - Número prefijado de sesiones. } \\
\text { - Menor número de sesiones. }\end{array}$ & $\begin{array}{l}\text { - Contenido flexible. } \\
\text { - Sin limitaciones prefijadas en el número de sesiones. } \\
\text { - Mayor número de sesiones. }\end{array}$ \\
\hline Seguimiento & - Largos (6-12 meses). & - Cortos o inexistentes. \\
\hline
\end{tabular}


aguda del trastorno y derivar al paciente al médico de atención primaria. De esta manera, se facilita la cronificación y el fenómeno de la puerta giratoria (Echeburúa, 2008).

\section{LAS NUEVAS TENDENCIAS Y LAS NUEVAS TERAPIAS PSICOLÓGICAS}

La terapia cognitivo-conductual (TCC) ha mostrado ser el desarrollo teórico más sólido disponible hasta la fecha y es la psicoterapia de referencia hoy por hoy (Roth y Fonagy, 2005). No ha habido progresos teóricos realmente significativos en las tres últimas décadas, pero se han acumulado estudios y datos fiables sobre su eficacia. Asimismo se han desarrollado las terapias en formatos grupales y se han aplicado a ellas también las nuevas tecnologías (programas de ordenador, realidad virtual, etcétera). Todo ello es positivo, siempre que no se desvirtúen los fundamentos mismos de la terapia cognitivo-conductual ni se minusvalore el énfasis necesario en la relación terapeuta-paciente o en el cumplimiento de las prescripciones terapéuticas. El lado negativo es el enfoque economicista aplicado a la terapia cognitivoconductual, que puede llevar a frivolizar este enfoque. Así, por ejemplo, en Gran Bretaña se están formando especialistas en TCC, especialmente en universidades privadas, a un nivel de formación profesional (primary care mental health workers) sin la cualificación requerida, que tratan de aplicar mecánicamente diversas técnicas terapéuticas (relajación, control de contingencias o restructuración cognitiva) y que constituyen una alternativa para atender a los pacientes con sintomatología ansioso-depresiva no grave que llegan a la Atención Primaria y que corren el peligro de colapsarla. Sin embargo, la mera formación en la aplicación de técnicas sin el conocimiento de los mecanismos de aprendizaje del comportamiento humano, tanto normal como anormal, así como sin la realización de una evaluación rigurosa que contemple el análisis funcional, parece una vía peligrosa.

En el ámbito de la terapia cognitivo-conductual, hay nuevos desarrollos que pueden resultar de gran interés en ciertos contextos, como el crecimiento personal postraumático (Tedeschi y Calhoun, 2004), basado en el aprendizaje a partir de los fracasos, o la entrevista motivacional (Miller y Rollnick, 1999), orientada a aquellos pacientes que no presentan una genuina motivación para el cambio (Pelechano, 2007). Otros enfoques resultan, sin embargo, más problemáticos. Así, por ejemplo, la terapia de aceptación y compromiso (ACT; Hayes, Follette y Linehan, 2004), considerada enfáticamente como la tercera ola en la terapia conductual (la primera fue el conductismo y la segunda el cognitivismo) no es propiamente una terapia psicológica. La ACT, que incorpora el yoga, la meditación zen, la relajación y la autohipnosis, es una terapia para alcanzar una vida más plena y cargada de sentido. Por ello, es una terapia que no se dirige a aliviar o eliminar los síntomas, sino a cosas más vagas (operativamente hablando), como recuperar una vida con sentido o aceptar la inevitabilidad del dolor o de los problemas. En general, los estudios llevados a cabo con la ACT y con la psicoterapia analítico funcional de Kohlenberg y Tsai (1991) hasta la fecha no son muy rigurosos metodológicamente y el tamaño del efecto obtenido ha sido moderado, por lo que no reúnen los requisitos exigidos para considerarlas como tratamientos empíricamente validados (Öst, 2008).

\section{LA DIFUSIÓN DE LOS TRATAMIENTOS PSICOLÓGICOS EFICACES}

No es exagerado afirmar que las terapias no validadas empíricamente se utilizan con más frecuencia que los tratamientos basados en la evidencia y que, por tanto, hay un desfase entre lo que se sabe y lo que se hace. Por chocante que pueda resultar, lo que parece hoy efectivo dista de estar disponible para la mayoría de la población. De este modo, hacer que lo útil sea utilizado se convierte en una prioridad de actuación (Echeburúa, 1998). La difusión no es algo que ocurre automáticamente, sino que está ligada a tres factores importantes: innovación (la aportación real de la nueva propuesta), canales de comunicación (el conocimiento de la nueva técnica por parte de los profesionales implicados) y transcurso del tiempo (necesario para romper con la resistencia al cambio en los 
profesionales) (Barlow, Levitt y Bufka, 1999; Rogers, 1995; Sobell, 1996). De hecho, los clínicos se muestran muy reticentes a cambiar líneas de actuación y formas de abordar los problemas que tienen sobreaprendidas (Persons, 1995; Wilson, 1996a).

La difusión de los tratamientos psicológicos eficaces depende, entre otras variables, del coste y de la duración de los mismos, así como de la existencia de manuales de tratamiento, que suelen ser bien aceptados por los clínicos (Addis, 1997; Wilson, 1996b).

Un problema de difusión importante es el relacionado con los tratamientos combinados, especialmente en los trastornos graves y de curso crónico. Permitásenos citar dos ejemplos concretos. Según estudios clínicos controlados rigurosos (Falloon, 1999; Hogarty et al., 1991), el tratamiento de la esquizofrenia debe llevarse a cabo con fármacos neurolépticos y con técnicas de rehabilitación psicosociales, que son las que garantizan una mayor prevención de las recaídas y un mejor ajuste social. Y, sin embargo, los pacientes psicóticos, que, en general, están bien tratados desde un punto de vista biológico (es decir, con antipsicóticos de última generación), no siempre se benefician de los tratamientos psicológicos, administrados de forma técnicamente correcta, que cuentan con una eficacia demostrada (Uriarte, 2000).

Un segundo ejemplo es el relacionado con el tratamiento del trastorno de déficit de atención con hiperactividad. Al margen del papel fundamental que desempeña en este trastorno la medicación (el metilfenidato, por ejemplo), el tratamiento cognitivo-conductual + fármaco, según el estudio más importante de seguimiento llevado a cabo hasta la fecha (MTA Cooperative Group 1999a, 1999b), resulta superior a la mera medicación porque añade, además de la reducción de síntomas, una mejora en la adquisición de habilidades y en la adaptación social. Sin embargo, los niños diagnosticados en la práctica clínica están habitualmente medicados, pero sólo una pequeña parte recibe un tratamiento cognitivo-conductual estructurado.

Por lo tanto, difundir entre los profesionales las terapias que resultan eficaces es una labor que todavía está por hacerse.

\section{CARACTERISTICAS PERSONALES DE LOS PSICÓLOGOS CLÍNICOS}

Por obvio que resulte decirlo, el psicólogo clínico va a estar en contacto con pacientes, es decir, con personas que sufren. Comprender al ser humano sufriente es el requisito previo para cualquier actuación profesional. Así, con independencia del ámbito concreto en el que se trabaje, los psicólogos clínicos deben aprender a observar (no sólo ver) y a escuchar (no sólo oír), así como a empatizar, comprender y analizar. Ésta es la única manera de comunicarse adecuadamente con otras personas y de ayudar a resolver los problemas planteados.

Realizar terapia psicológica no es sólo una mera aplicación de técnicas, sino que requiere previamente de habilidades para establecer una relación que sirva para aliviar el sufrimiento, así como de habilidades para obtener información, ordenarla y devolverla al paciente, de modo que le permita una comprensión de las causas y el mantenimiento de sus problemas. Sólo posteriormente se puede pasar a la aplicación de técnicas que conduzcan a la modificación de pautas de comportamiento, cognitivas y emocionales. Por lo tanto, lo terapéutico no es sólo la técnica, sino también la relación, el modo de evaluar, el modo de explicar los problemas y el modo de realizar nuevos aprendizajes.

Más allá de unas destrezas profesionales, se requieren en el psicólogo clínico unas características personales que faciliten el establecimiento de una alianza terapéutica con el paciente. Por ello, no se puede desatender el peso específico de las características personales del psicólogo clínico en el resultado final de la terapia. Ante la constatación empírica de que hay una gran variabilidad en los logros obtenidos de unos terapeutas a otros, al margen de las técnicas terapéuticas utilizadas, el perfil personal idóneo del psicólogo clínico requiere estudios más precisos. Hay, sin embargo, algunas características básicas, ya conocidas, que facilitan la alianza terapéutica: equilibrio y control emocional, sentido común, capacidad de empatía, ausencia de rigidez y ganas genuinas de prestar ayuda. Todo ello facilita que tengan una actitud positiva ante la formación continuada y estén receptivos ante el progreso del conocimiento (Echeburúa, 2008; Echeburúa y Corral, 2001). 
Pero, además, los psicólogos clínicos deben contar con unas habilidades interpersonales que faciliten el trabajo en equipo o, al menos, el contacto con otros profesionales. Si los psicólogos clínicos están integrados en un centro de salud mental o en un hospital, tienen que saber trabajar en equipo. Ello implica interactuar con otros profesionales de la salud (médicos de atención primaria, psiquiatras o enfermeras), lo que obliga a saber utilizar un lenguaje común, a ser flexibles, a tener unas habilidades de comunicación, a marcarse objetivos concretos y a contar con un modelo integrador de salud. Y si los psicólogos clínicos trabajan en la consulta privada, deben tener estas mismas habilidades para relacionarse con otros colegas, derivar casos a otros profesionales o saber comunicarse adecuadamente con los familiares de los pacientes.

En resumen, no actúa necesariamente mejor con los pacientes el psicólogo clínico que más teoría sabe o que más experiencia acumula. Sin olvidar otras claves esenciales (conocimientos profesionales y disposición para estar al día, facilidad para adaptarse a los cambios, capacidad para integrarse en un equipo o motivación por la profesión), la inteligencia emocional, la autoestima apropiada y las habilidades interpersonales constituyen una herramienta fundamental para el desempeño adecuado de la psicología clínica.

\section{PROBLEMAS DEONTOLÓGICOS Y PRÁCTICA INADECUADA}

Hoy en día se requiere prestar una atención mayor a los aspectos éticos implicados en el quehacer profesional, especialmente a todo lo referido a la vulneración del derecho a la intimidad (es decir, a la ruptura del secreto profesional), a la mala praxis y a los informes periciales a petición de parte (Del Río, 2005; Echeburúa, 2002).

Según el Artículo 4.7b de la LOPS, de aplicación para los psicólogos clínicos, la actuación del profesional sanitario «estará basada en la evidencia científica y en los medios disponibles y amparados en guías y protocolos de práctica clínica y asistencial». Ello quiere decir, en un plano le- gal, que la evaluación de las terapias psicológicas permite establecer operativamente la práctica profesional inadecuada, que da lugar a hipotéticas responsabilidades civiles o penales.

En concreto, la mala praxis se configura cuando un profesional se separa de la lex artis (normas o guías de actuación profesional consensuadas) y actúa de forma técnicamente inadecuada. Esto es más claro cuando, como ocurre en la actualidad, comienzan a existir guías de actuación profesional en los distintos trastornos (por ejemplo, en nuestro contexto, Pérez et al., 2003).

Los psiquiatras han sido acusados por práctica inadecuada principalmente por causa de diagnósticos erróneos, certificación de hospitalización impropia, suicidio del paciente, efectos dañinos de la terapia electroconvulsiva y de los psicofármacos (desarrollo de discinesia tardía con los neurolépticos), divulgación impropia de información y relaciones sexuales con los pacientes. Pero también se puede demandar a un psicólogo clínico por algunos de estos puntos o por tratar sólo con psicoterapia a un paciente con un trastorno bipolar, sin que se le derive también al psiquiatra.

Cuando los pacientes van a formar parte de una investigación clínica, es preciso contar con la aprobación del Comité de Ética del Hospital o de la universidad y, a nivel individual, con el consentimiento informado de cada uno de los pacientes. En cualquier caso, hay que garantizar la confidencialidad y el archivo de las historias, así como el acceso que pueden tener los pacientes a sus historias clínicas y a los resultados del estudio.

\section{CONCLUSIONES}

La integración de las terapias psicológicas en el Sistema Nacional de Salud exige la evaluación rigurosa de las mismas (Barlow y Hoffman, 1997). Por lo que a los instrumentos de evaluación se refiere, las entrevistas estructuradas, las escalas de evaluación heteroaplicadas y los autoinformes - cada vez más breves, específicos y sensibles al cambio terapéuticovan a constituir los pilares del diagnóstico clínico al servicio de esta tarea evaluadora.

Las investigaciones deben llevarse a cabo, fundamentalmente, en los centros de salud men- 
tal o en los centros de atención primaria porque los resultados obtenidos en estos ámbitos son más fácilmente generalizables que los conseguidos en universidades, en la práctica privada o con voluntarios. Pero las investigaciones en el marco de la sanidad pública no siempre pueden ser controladas y a veces deben limitarse a estudios naturalistas o abiertos. Al margen de los problemas metodológicos suscitados (falta de un grupo de control, tratamiento flexible, farmacoterapia no controlada, etcétera), este tipo de investigaciones puede representar una evaluación rigurosa del tipo de tratamiento ofertado (Westbrook y Kirk, 2005).

Al margen de las limitaciones de las investigaciones clínicas, éstas van a suponer - en realidad, han supuesto ya - un camino sin retorno en la práctica profesional de la psicología clínica. Por ello, las terapias psicológicas van a formar parte de intervenciones breves (10-20 sesiones) y específicas y van a estar estandarizadas, es decir, con una descripción precisa de los instrumentos de evaluación, el programa de tratamiento detallado y el formato de aplicación (individual o grupal), lo que no es incompatible con la necesaria flexibilidad en la aplicación de estos tratamientos en función de las peculiaridades concretas de cada paciente (Salaberría, Sánchez y Corral, 2009; Van Hasselt y Hersen, 1996).

Otra línea sugerente, en un intento de tomar en consideración las diferencias individuales de los pacientes, es asignar tratamientos distintos a tipos de pacientes específicos en el ámbito de un mismo trastorno - el denominado emparejamiento paciente-tratamiento-, como ya se ha empezado a hacer en el estudio del alcoholismo (Allen y Kaden, 1995). Asimismo el control de la relación terapeuta-paciente es otra línea de investigación atractiva, especialmente en los trastornos caracterizados por una negación del problema. Así, por ejemplo, en la terapia conductual dialéctica de Linehan (1993) con el trastorno límite de la personalidad, se concede una gran importancia al establecimiento de una relación empática, no directiva y de aceptación como paso previo para el establecimiento de las técnicas específicas de intervención.

Hay una superioridad clara de la terapia cognitivo-conductual sobre otro tipo de tratamientos (terapia psicodinámica, terapia sistémica, enfoque humanista y terapia ecléctica) en una gran variedad de trastornos (Seligman, 1998). El grado de conocimiento sobre la efectividad de las terapias dinámicas, sistémicas y existenciales-humanistas en diferentes trastornos es desconocido en muchos casos. Sería conveniente que los terapeutas e investigadores que trabajan desde estos modelos pudieran aportar datos que podrían resultar de interés. Algunas de estas orientaciones clínicas (sistémicas, interpersonales, etcétera) pueden ser útiles en la medida en que incorporan técnicas procedentes del enfoque cognitivo-conductual. Ello no obsta para que haya cuadros clínicos, como las psicosis, los problemas somatomorfos y los trastornos de personalidad, en que los resultados obtenidos sean aún escasos. En este sentido los fracasos y abandonos terapéuticos, derivados de fuentes diversas (errores del terapeuta, variables del paciente, complejidad del cuadro clínico, etcétera), deben ser una vía de estudio, no suficientemente explorada en la actualidad.

La tendencia actual es a la búsqueda de programas de tratamiento estandarizados, centrados en la solución de problemas concretos del aquí y ahora, más allá de las nebulosas psicoterapias basadas en la escucha, el apoyo y el inútil buceo en la desgraciada infancia del paciente. De este modo, lo que puede explicar la eficacia similar de la terapia cognitiva y la terapia interpersonal en el tratamiento de la depresión es el acento puesto por una y otra en la estrategia de solución de problemas (Marks, 1992).

Asimismo es sugerente la investigación reciente sobre el efecto potenciador de los tratamientos combinados (terapia cognitivo-conductual + psicofármacos) en diferentes cuadros clínicos (Echeburúa et al., 1998; Labrador et al., 2000). Los fármacos pueden actuar sobre la reducción de síntomas; los tratamientos psicológicos, sobre el aumento de competencias. Se trata, en último término, de aprovechar, por un lado, la potencialidad terapéutica de los psicofármacos a corto plazo para conseguir cambios de conducta rápidos, motivar al paciente al tratamiento y ponerle en disposición conductual y cognitiva de asumir las tareas propuestas por la terapia psicológica; por otro, de hacer desaparecer paulatinamente los fármacos y de integrar el tratamiento psicológico. De este modo, el paciente puede atribuir los logros terapéuticos a 
sí mismo y evitar así la dependencia psicológica de los fármacos y del terapeuta. Quedan aún, sin embargo, por determinar los cuadros clínicos tributarios de estos tratamientos combinados y los parámetros significativos implicados en la interacción (dosis, duración, desvanecimiento gradual, etcétera) (Nathan y Gorman, 1998).

Conviene hacer una autocrítica, a modo de reflexión ante el futuro inmediato. A un nivel teórico, la coherencia inicial postulada entre la teoría y la práctica se ha debilitado a expensas de una atención selectiva a la utilidad de la intervención terapéutica. El énfasis actual en el carácter tecnológico de las terapias psicológicas ha llevado en muchos casos a minimizar el papel de la teoría: ha habido un tránsito de la ciencia a la tecnología y un canto al pragmatismo clínico. De hecho, la evidencia experimental se ha referido especialmente a la comprobación de la eficacia de las técnicas terapéuticas, pero no así a la verificación de los principios teóricos en que supuestamente se basan (Echeburúa, 1998). Y a un nivel práctico, los enfoques cognitivo-conductuales, aun siendo los más efectivos, distan aún mucho de resolver problemas clínicos significativos: entre otros, el incumplimiento de las prescripciones terapéuticas; la falta de motivación para el tratamiento en algunos trastornos (adicciones, parafilias o conductas violentas); o la terapia de los cuadros clínicos más graves de la psiquiatría pesada (psicosis o trastorno bipolar). Y, además, la mejoría lograda en otros trastornos no siempre se corresponde con una calidad de vida similar a la de las personas normales. Todo ello es un reto para la investigación futura.

Por último, la psicología clínica basada en la evidencia es un camino irreversible. Pero todavía hay muchos cuadros clínicos sobre los que no hay tratamientos con una evidencia inequívoca. Por ello, la afirmación de que no hay pruebas sobre la eficacia de un determinado tratamiento no debe ser tomada como que existen pruebas de su ineficacia.

\section{REFERENCIAS}

Addis, M.E. (1997). Evaluating the treatment manual as a means of disseminating empirically validated psy- chotherapies. Clinical Psychology: Science and Practice, 4, 1-11.

Allen, J.P. y Kadden, R.M. (1995). Matching clients to alcohol treatment. En R.K. Hester y W.R. Miller (eds.). Handbook of alcoholism treatment approaches. Effective alternatives (2nd. ed.). Boston. Allyn and Bacon.

Bados, A., García, E. y Fuste, A. (2002). Eficacia y utilidad clínica de la terapia psicológica. Revista Internacional de Psicología Clínica y de la Salud, 2, 477-502.

Barlow, D.H. (1994). Psychological interventions in the era of managed competition. Clinical Psychology: Science and Practice, 1, 109-123.

Barlow, D.H. y Hoffman, S.G. (1997). Efficacy and dissemination of psychological treatments. En D.M. Clark y C.G. Fairburn (eds.). Science and practice of cognitive behavior therapy. Oxford. Oxford University Press.

Barlow, D.H., Levitt, J.T. y Bufka, L.F. (1999). The dissemination of empirically supported treatments: a view to the future. Behaviour Research and Therapy, 37, 147-162.

Bayés, R. (1984). ¿Por qué funcionan las terapias comportamentales? Anuario de Psicología, 30-31, 127-147.

Becoña, E. (1999). La discrepancia entre la investigación y la práctica clínica de la terapia de conducta. Revista de Psicopatología y Psicología Clínica, 4, 71-103.

Becoña, E., Vázquez. M.J., Míguez, M.C. et al. (2004). Guías de tratamiento y guías para la práctica clínica psicológica. Papeles del Psicólogo, 87, 9-19.

Chambless, D.L. (1996). In defense of dissemination of empirically supported psychological interventions. Clinical Psychology: Science and Practice, 3, 230-235.

Chambless, D.L. y Hollon, S.D. (1998). Defining empirically supported therapies. Journal of Consulting and Clinical Psychology, 66, 3-18.

Chambless, D.L., Sanderson, W.C., Shoham, V. et al. (1996). An update on empirically validated therapies. The Clinical Psychologist, 49, 5-18.

Chambless, D.L., Baker, M.J., Baucom, D.H. et al. (1998). Update on empirically validated therapies: II. The Clinical Psychologist, 51, 3-15.

Del Río, C. (2005). Guía de ética profesional en psicología clínica. Madrid. Pirámide.

Echeburúa, E. (1998). ¿Qué terapias psicológicas son eficaces? Un reto ante el 2000. Revista de Psicopatología y Psicología Clínica, 3, 149-160.

Echeburúa, E. (2002). El secreto profesional en la práctica de la psicología clínica y forense: Alcance y límites de la confidencialidad. Análisis y Modificación de Conducta, 28, 498-504.

Echeburúa, E. (2008). Retos de futuro de la psicología clínica. Análisis y Modificación de Conducta, 34, 209-207.

Echeburúa, E. y Corral, P. (2001). Eficacia de las terapias psicológicas: de la investigación a la práctica clínica. Revista Internacional de Psicología Clínica y de la Salud, 1, 181-204. 
Echeburúa, E., Corral, P. y Salaberría, K. (1998). Terapia de conducta y tratamientos psicofarmacológicos. En M.A. Vallejo (Ed.). Manual de terapia de conducta (vol. 1). Madrid: Dykinson.

Echeburúa, E., Corral, P. y Salaberría, K. (2005). Reflexiones ante la formación de los psicólogos. Retos de futuro. Análisis y Modificación de Conducta, 31, 175-188.

Falloon, R.H. (1999). Optimal treatment for psychosis in an international multisite demonstration project. Psychiatric Services, 50, 615-618.

Gabbard, G.O., Lazar, S.G., Hornberger, J. y Spiegel, D. (1997). The economic impacto of psychotherapy: A review. American Journal of Psychiatry, 154, 147-155.

Gambrill, E. (1999). Evidence-based clinical behavior analysis, evidence-based medicine and the Cochrane Collaboration. Journal of Behavior Therapy and Experimental Psychiatry, 30, 1-14.

Guimón, J. (2004). Eficacia de las psicoterapias en salud mental. Bilbao: Desclée de Brouwer.

Hayes, S.C., Follette, V.M. y Linehan, M.M. (2004). Mindfulness and acceptance: expanding the cognitive behavioural tradition. New York: Guilford Press.

Hickey, P. (1998). DSM and behavior therapy. The Behavior Therapist, March 1998, 43-46.

Hogarty, G.E., Anderson, C.M., Reiss, D.J. et al. (1991). Family psychoeducation, social skills training, and maintenance chemotherapy in the aftercare treatment of schizophrenia: II. Two-year effects of a controlled study on relapse and adjustment. Archives of General Psychiatry, 48, 340-347.

Howard, K.I., Krause, M.S. y Orlinsky, D.E. (1986). The dose-effect relationship in psychotherapy. American Psychologist, 41, 159-164.

Kohlenberg, R.J. y Tsai, M. (1991). Functional analytic psychotherapy. Creating intense and curative therapeutic relationship. New York: Plenum Press.

Labrador, F.J., Echeburúa, E. y Becoña, E. (2000). Guía de tratamientos psicológicos. Madrid: Dykinson.

Layard, R. (2006). The case for psychological treatment centres. British Medical Journal, 332, 1030-1032.

Leichsenring, F. y Leibing, E. (2003). The effectiveness of psychodynamic therapy and cognitive behavior therapy in the treatment of personality disorders: a metaanalysis. American Journal of Psychiatry, 160, 12231232.

Linehan, M.M. (1993). Cognitive-behavioral treatment of borderline personality disorders. New York: Guilford Press.

Mace, C., Moorey, S. y Roberts, B. (2005). Guía práctica de terapias psicológicas. Guía crítica para los clínicos. Barcelona: Ariel.

Marks, I.M. (1992). Behavioural psychotherapy towards the millenium. En J. Cottraux, P. Legeron y E. Mollard (eds.). Which psychotherapies in year 2000?. Amsterdam. Swets and Zeitlinger.
Marks, I.M. y O’Sullivan, G. (1992). Psicofármacos y tratamientos psicológicos en la agorafobia/pánico y en el trastorno obsesivo-compulsivo. En E. Echeburúa (Ed.). Avances en el tratamiento psicológico de los trastornos de ansiedad. Madrid: Pirámide.

Miller, W.R. y Rollnick, S. (1999). La entrevista motivacional. Preparar para el cambio de conductas adictivas. Barcelona: Paidós.

MTA Cooperative Group (1999a). A 14-month randomized clinical trial of treatment strategies for attentiondeficit/hiperactivity disorder. Archives of General Psychiatry, 56, 1073-1086.

MTA Cooperative Group (1999b). Moderators and mediators of treatment response for children with attentiondeficit/hiperactivity disorder. Archives of General Psychiatry, 56, 1088-1096.

Nathan, P.E. y Gorman, J.M. (eds.) (1998). A guide to treatments that work. Oxford: Oxford University Press.

Öst, L.G. (2008). Efficacy of the third wave of behavioral therapies: A systematic review and meta-analysis. $\mathrm{Be}$ haviour Research and Therapy, 46, 296-321.

Pascual, J., Frías, M.D. y Monterde, H. (2004). Tratamientos psicológicos con apoyo empírico y práctica clínica basada en la evidencia. Papeles del Psicólogo, 87, 1-8.

Pelechano, V. (2007). Viejas y nuevas cuestiones en las viejas y nuevas terapias psicológicas. Revista de Psicopatología y Psicología Clínica, 12, 71-90.

Pérez, M., Fernández-Hermida, J.R., Fernández, C. y Amigo, I. (Eds.) (2003). Guía de tratamientos psicológicos eficaces. Madrid: Pirámide.

Persons, J.B. (1995). Why practicing psychologists are slow to adopt empirically validated treatments. En S.C. Hayes, V.M. Follette, R.M. Dawes y K.E. Grady (eds.). Scientific standards of psychological practice. Issues and recommendations. Reno, Nevada: Context Press.

Rogers, E.M. (1995). Lessons for guidelines from the diffussion of innovation. Commisssions Journal of Quality Improvements, 21, 325-328.

Roth, A. y Fonagy, P. (2005). What works for whom? A critical review of psychotherapy research $\left(2^{\text {nd }} \mathrm{ed}\right)$. New York: Guilford Press.

Salaberría, K., Páez, D. y Echeburúa, E. (1996). Evaluación de la validez del cambio inducido por los tratamientos psicológicos: una propuesta metodológica. Boletín de Psicología, 52, 71-96.

Salaberría, K., Sánchez, A. y Corral, P. (2009). Eficacia de un programa de apoyo psicológico a inmigrantes: un estudio de casos. Revista de Psicopatología y Psicología Clínica, 14, 153-164.

Seligman, M.E.P. (1995). The effectiveness of psychotherapy. The Consumer Reports Study. American Psychologist, 50, 965-974.

Seligman, M.E.P. (1998). Foreword-A purpose. En P.E. Nathan y J.M. Gorman, J.M. (Eds.). A guide to treatments that work. Oxford: Oxford University Press. 
Smith, M.L., Glass, G.V. y Miller, T.I. (1980). The benefits of psychotherapy. Baltimore, Maryland: John Hopkins University Press.

Sobell, L.C. (1996). Bridging the gap between scientists and practitioners: The challenge before us. Behavior Therapy, 27, 297-320.

Task Force on Promotion and Dissemination of Psychological Procedures (1995). Training and dissemination of empirically-validated psychological treatments: Report and recommendations. The Clinical Psychologist, 48, 3-29.

Tedeschi, R.G. y Calhoun, L.G. (2004). A clinical approach to posttraumatic growth. En P.A. Linley y S. Joseph (Eds.). Positive psychology in practice. Hoboken, NJ: John Wiley \& Sons.

Turner, S.M., Beidel, D.C., Spaulding, S.A. y Brown, J.M. (1995). The practice of behavior therapy: A national survey of cost and methods. The Behavior Therapist, $18,1-4$.

Uriarte, J.J. (2000). Editorial: Psiquiatría social. THMNews, 53, 1-2.

Van Hasselt, V.B. y Hersen, M. (eds.) (1996). Sourcebook of psychological treatments manuals for adult disorders. New York: Plenum Press.

Westbrook, D. y Kirk, J. (2005). The clinical effectiveness of cognitive behaviour therapy: outcome for a large sample of adults treated in routine practice. Behaviour Research and Therapy, 43, 1243-1261.

Wilson, G.T. (1996a). Empirically validated treatments: Reality and resistence. Clinical Psychology: Science and Practice, 34, 241-244.

Wilson, G.T. (1996b). Manual-based treatments: the clinical application of research findings. Behaviour Research and Therapy, 34, 295-314. 